\title{
POSITIVE SOLUTIONS OF SUBLINEAR ELLIPTIC BOUNDARY VALUE PROBLEMS
}

\author{
Kazuaki Taira and Kenichiro UmezU
}

\section{Introduction AND Results}

This paper is a continuation of the previous papers Taira-Umezu [TU1] and [TU2] where we studied global static bifurcation theory for a class of degenerate boundary value problems for nonlinear second-order elliptic differential operators. The previous papers treated the asymptotic linear and nonlinear cases, for example, such nonlinear terms as $u+1 / u$ and $u^{p}, p>1$, near $u=+\infty$, by using the Leray-Schauder degree theory. The purpose of this paper is to study more general nonlinear terms such as $\sqrt{u}, \log (1+u)$ and $e^{-u}$, and is to prove the existence and uniqueness of positive solutions of nonlinear elliptic boundary value problems, by making good use of the super-subsolution method. We remark that the variational method would break down, since our boundary condition is degenerate.

Let $D$ be a bounded domain of Euclidean space $\mathbf{R}^{N}, N \geq 2$, with $C^{\infty}$ boundary $\partial D$; its closure $\bar{D}=D \cup \partial D$ is an $N$-dimensional, compact $C^{\infty}$ manifold with boundary. We let

$$
A u(x)=-\sum_{i=1}^{N} \frac{\partial}{\partial x_{i}}\left(\sum_{j=1}^{N} a^{i j}(x) \frac{\partial u}{\partial x_{j}}(x)\right)+c(x) u(x)
$$

be a second-order, elliptic differential operator with real $C^{\infty}$ coefficients on $\bar{D}$ such that:

(1) $a^{i j}(x)=a^{j i}(x), x \in \bar{D}, 1 \leq i, j \leq N$, and there exists a constant $a_{0}>0$ such that

$$
\sum_{i, j=1}^{N} a^{i j}(x) \xi_{i} \xi_{j} \geq a_{0}|\xi|^{2}, x \in \bar{D}, \xi \in \mathbf{R}^{N} .
$$

(2) $c(x) \geq 0$ on $\bar{D}$.

In this paper we consider the following general nonlinear elliptic boundary value problem: Given function $f(x, \xi)$ defined on $\bar{D} \times[0, \infty)$, find a nonnegative function $u$ in $D$ such that

$$
\begin{cases}A u=f(x, u) & \text { in } D, \\ B u=a \frac{\partial u}{\partial \nu}+b u=0 & \text { on } \partial D .\end{cases}
$$

Here:

1991 Mathematics Subject Classification. 35J65, 35P30, 35J25. 
(1) $a \in C^{\infty}(\partial D)$ and $a \geq 0$ on $\partial D$.

(2) $b \in C^{\infty}(\partial D)$ and $b \geq 0$ on $\partial D$.

(3) $\partial / \partial \boldsymbol{\nu}$ is the conormal derivative associated with the operator $A: \partial / \partial \boldsymbol{\nu}=$ $\sum_{i, j=1}^{N} a^{i j} n_{j} \partial / \partial x_{i}$, where $\mathbf{n}=\left(n_{1}, n_{2}, \cdots, n_{N}\right)$ is the unit exterior normal to the boundary $\partial D$.

First we state our fundamental hypotheses on the functions $a, b$ and $c$ :

(H.1) $b\left(x^{\prime}\right)>0$ on $M=\left\{x^{\prime} \in \partial D: a\left(x^{\prime}\right)=0\right\}$.

(H.2) $c(x)>0$ in $D$.

It is worth pointing out here that the boundary condition $B$ is non-degenerate if and only if either $a \neq 0$ on $\partial D$ or $a \equiv 0$ and $b \neq 0$ on $\partial D$. In particular, if $a \equiv 1$ and $b \equiv 0$ on $\partial D$ (resp. $a \equiv 0$ and $b \equiv 1$ on $\partial D$ ), then the boundary condition $B$ is the so-called Neumann (resp. Dirichlet) condition.

A solution $u \in C^{2}(\bar{D})$ of problem $(*)$ is said to be nontrivial if it does not identically equal zero on $\bar{D}$. We call a nontrivial solution $u$ of problem $(*)$ a positive solution if $u(x) \geq 0$ on $\bar{D}$.

Let $\lambda_{1}$ be the first eigenvalue of the linearized boundary value problem

$$
\begin{cases}A u=\lambda u & \text { in } D, \\ B u=0 & \text { on } \partial D .\end{cases}
$$

By [Ta2, Theorem 1], we know that the eigenvalue $\lambda_{1}$ is positive and simple with positive eigenfunction in $D$.

Our existence theorem for positive solutions of problem $(*)$ is stated as follows (cf. [BO, Theorem 2]):

Theorem 1. Assume that hypotheses (H.1) and (H.2) are satisfied and that the function $f(x, \xi)$ belongs to $C^{\theta}(\bar{D} \times[0, \sigma]), 0<\theta<1$, for every $\sigma>0$, and satisfies the slope condition: For every $\sigma>0$, there exists a constant $\omega=\omega(\sigma)>0$, independent of $x \in \bar{D}$, such that

$$
f(x, \xi)-f(x, \eta)>-\omega(\xi-\eta), \quad x \in \bar{D}, 0 \leq \eta<\xi \leq \sigma .
$$

If in addition the two limits

$$
\ell(x)=\lim _{\xi \downarrow 0} \frac{f(x, \xi)}{\xi}
$$

and

$$
m(x)=\lim _{\xi \rightarrow \infty} \frac{f(x, \xi)}{\xi}
$$

exist uniformly for all $x \in \bar{D}$ and if we have

$$
m(x)<\lambda_{1}<\ell(x), \quad x \in \bar{D},
$$

then problem $(*)$ has a positive solution $u \in C^{2+\theta}(\bar{D})$.

If the nonlinear term $f(x, \xi)$ is independent of $x$, then we can prove that condition (1.1) is necessary and sufficient for the existence of positive solutions of problem $(*)$; more precisely, we have the following generalization of [BO, Theorem 1] to the degenerate case: 
Theorem 2. Assume that hypotheses (H.1) and (H.2) are satisfied, and that the function $f(x, \xi)=f(\xi)$ is independent of $x \in \bar{D}$ and further that the function $f(\xi) / \xi$ is strictly decreasing for $0<\xi<\infty$. We let

$$
\ell=\lim _{\xi \downarrow 0} \frac{f(\xi)}{\xi}, \quad m=\lim _{\xi \rightarrow \infty} \frac{f(\xi)}{\xi} .
$$

Then problem $(*)$ has a positive solution $u \in C^{2+\theta}(\bar{D})$ if and only if

$$
m<\lambda_{1}<\ell \text {. }
$$

Furthermore, the solution $u$ is unique in the space $C^{2}(\bar{D})$.

Now, as an application of Theorem 2, we consider global static bifurcation problems for the following semilinear elliptic boundary value problem:

$$
\begin{cases}A u-\lambda u+h(u)=0 & \text { in } D, \\ B u=0 & \text { on } \partial D .\end{cases}
$$

The next corollary, which is an immediate consequence of Theorem 2, allows us to treat more general nonlinear terms $h$ than [TU1, Theorem 1] as is shown in Examples 1-4 below.

Corollary 1. Assume that $h(\xi)$ is a function in $C^{\theta}([0, \sigma]), 0<\theta<1$, for every $\sigma>0$, and that the function $h(\xi) / \xi$ is strictly increasing for $0<\xi<\infty$. We let

$$
\alpha=\lim _{\xi \downarrow 0} \frac{h(\xi)}{\xi}, \quad \beta=\lim _{\xi \rightarrow \infty} \frac{h(\xi)}{\xi} .
$$

Then problem $(* *)$ has a unique positive solution $u \in C^{2+\theta}(\bar{D})$ if and only if $\lambda_{1}+$ $\alpha<\lambda<\lambda_{1}+\beta$.

For Corollary 1, we give four simple examples of the function $h(\xi)$ :

Example 1. $h(\xi)=(k / 6) \xi^{3}$ for $0 \leq \xi \leq 1$ and $h(\xi)=k(\xi+1 /(2 \xi)-4 / 3)$ for $1<\xi<\infty$, where $k$ is a positive constant. In this case, $\alpha=0, \beta=k$ and so $\lambda_{1}<\lambda<\lambda_{1}+k$.

Example 2. $h(\xi)=\xi^{p}, p>1$. In this case, $\alpha=0, \beta=\infty$ and so $\lambda_{1}<\lambda<\infty$.

Example 3. $h(\xi)=-\sqrt{\xi}$. In this case, $\alpha=-\infty, \beta=0$ and so $-\infty<\lambda<\lambda_{1}$.

Example 4. $h(\xi)=-e^{-\xi}$. In this case, $\alpha=-\infty, \beta=0$ and so $-\infty<\lambda<\lambda_{1}$.

Similarly, we consider the following semilinear elliptic eigenvalue problem:

$$
(* * *) \quad \begin{cases}A u-\lambda g(u)=0 & \text { in } D, \\ B u=0 & \text { on } \partial D .\end{cases}
$$

Then we have the following generalization of [SC, Theorem 2.1] to the degenerate case: 
Corollary 2. Assume that $g(\xi)$ is a function in $C^{\theta}([0, \sigma]), 0<\theta<1$, for every $\sigma>0$, and that the function $g(\xi) / \xi$ is strictly decreasing for $0<\xi<\infty$. We let

$$
\gamma=\lim _{\xi \downarrow 0} \frac{g(\xi)}{\xi}, \quad \delta=\lim _{\xi \rightarrow \infty} \frac{g(\xi)}{\xi}
$$

Then problem $(* * *)$ has a unique positive solution $u \in C^{2+\theta}(\bar{D})$ if and only if $\lambda_{1} / \gamma<\lambda<\lambda_{1} / \delta$.

For Corollary 2, we give three simple examples of the function $g(\xi)$ :

Example 5. $g(\xi)=\sqrt{\xi}$. In this case, $\gamma=\infty, \delta=0$ and so $0<\lambda<\infty$.

Example 6. $g(\xi)=e^{-\xi}$. In this case, $\gamma=\infty, \delta=0$ and so $0<\lambda<\infty$.

Example 7. $g(\xi)=\log (1+\xi)$. In this case, $\gamma=1, \delta=0$ and so $\lambda_{1}<\lambda<\infty$.

\section{Proof of Theorem 1}

Our proof of Theorem 1 is carried out by making use of the super-subsolution method just as in the proof of [De, Theorem 2.2].

A nonnegative function $\psi \in C^{2}(\bar{D})$ is said to be a supersolution of problem $(*)$ if it satisfies the conditions:

$$
\begin{cases}A \psi-f(x, \psi) \geq 0 & \text { in } D \\ B \psi \geq 0 & \text { on } \partial D .\end{cases}
$$

Similarly, a nonnegative function $\phi \in C^{2}(\bar{D})$ is said to be a subsolution of problem $(*)$ if it satisfies the conditions:

$$
\begin{cases}A \phi-f(x, \phi) \leq 0 & \text { in } D \\ B \phi \leq 0 & \text { on } \partial D\end{cases}
$$

(I) First we construct a subsolution of problem $(*)$.

By condition (1.1), we can find a constant $c_{1}>0$ such that

$$
f(x, \xi) \geq \lambda_{1} \xi, \quad x \in \bar{D}, 0<\xi<c_{1} .
$$

On the other hand, it is known (cf. [Ta2, Theorem 1]) that the linearized boundary value problem

$$
\begin{cases}A \varphi=\lambda_{1} \varphi & \text { in } D, \\ B \varphi=0 & \text { on } \partial D\end{cases}
$$

has a positive eigenfunction $\varphi \in C^{2+\theta}(\bar{D})$. If we let

$$
\phi_{\varepsilon}=\varepsilon \varphi
$$

for $\varepsilon>0$ sufficiently small, we may assume that

$$
\max _{\bar{D}} \phi_{\varepsilon}<c_{1} .
$$


Then we have by condition (2.1)

$$
\begin{cases}A \phi_{\varepsilon}-f\left(x, \phi_{\varepsilon}\right) \leq \lambda_{1} \phi_{\varepsilon}-\lambda_{1} \phi_{\varepsilon}=0 & \text { in } D, \\ B \phi_{\varepsilon}=0 & \text { on } \partial D .\end{cases}
$$

This proves that the function $\phi_{\varepsilon} \in C^{2+\theta}(\bar{D})$ is a subsolution of problem $(*)$.

(II) In order to construct a supersolution of problem $(*)$, we make use of the theory of positive operators in ordered Banach spaces (cf. [Am]).

A Banach space $X$ is called an ordered Banach space if it is an ordered set. For an ordered Banach space $X$ having the ordering $\leq$, the set $Q=\{x \in X: x \geq 0\}$ is called the positive cone in $X$.

For functions $u$ and $v$ in $C(\bar{D})$, we write $u \leq v$ if $u(x) \leq v(x)$ for all $x \in \bar{D}$. Then the space $C(\bar{D})$ becomes an ordered Banach space with the ordering $\leq$. Moreover, if we let

$$
P=\{u \in C(\bar{D}): u \geq 0\},
$$

then the set $P$ is the positive cone in $C(\bar{D})$.

By [TU2, Theorem 1.1], we can introduce a continuous linear operator

$$
K: C^{\theta}(\bar{D}) \longrightarrow C^{2+\theta}(\bar{D})
$$

as follows: For any $v \in C^{\theta}(\bar{D})$, the function $u=K v \in C^{2+\theta}(\bar{D})$ is the unique solution of the boundary value problem

$$
\begin{cases}A u=v & \text { in } D, \\ B u=0 & \text { on } \partial D .\end{cases}
$$

Now we introduce an ordered Banach subspace of $C(\bar{D})$ which is associated with the resolvent $K$.

We let

$$
e=K 1 \in C^{2+\theta}(\bar{D})
$$

and

$$
C_{e}(\bar{D})=\{u \in C(\bar{D}) \text { : there is a constant } c>0 \text { such that }-c e \leq u \leq c e\} .
$$

Then the space $C_{e}(\bar{D})$ is given a norm by the formula

$$
\|u\|_{e}=\inf \{c>0:-c e \leq u \leq c e\} .
$$

If we let

$$
P_{e}=\left\{u \in C_{e}(\bar{D}): u \geq 0\right\},
$$

it is easy to verify that the space $C_{e}(\bar{D})$ is an ordered Banach space having the positive cone $P_{e}$ with nonempty interior. Moreover, by [TU1, Proposition 2.2], we can extend uniquely the resolvent $K$ to a strongly positive, compact linear operator

$$
K: C_{e}(\bar{D}) \longrightarrow C_{e}(\bar{D}) .
$$

Here we recall that $K$ is said to be strongly positive if $v \in P_{e}$ and $v \not \equiv 0$ on $\bar{D}$, then the function $K v$ is an interior point of $P_{e}$.

The next lemma plays an important role in the construction of a supersolution of problem $(*)$ (cf. [Kr, Theorem 2.16]): 
Lemma (The positivity lemma). Let $T: C_{e}(\bar{D}) \rightarrow C_{e}(\bar{D})$ be a strongly positive, compact linear operator and $\lambda_{0}$ the largest eigenvalue of $T$. Then, for any given positive function $g \in C_{e}(\bar{D})$, the equation

$$
\lambda v-T v=g
$$

has a unique positive solution $v \in C_{e}(\bar{D})$ for each $\lambda>\lambda_{0}$.

(III) By condition (1.1), we can find constants $c_{2}>0$ and $0<d<\lambda_{1}$ such that

$$
f(x, \xi) \leq\left(\lambda_{1}-d\right) \xi, \quad x \in \bar{D}, \xi>c_{2} .
$$

Hence, if we let

$$
k=\max \left\{|f(x, \xi)|: x \in \bar{D}, 0 \leq \xi \leq c_{2}\right\}
$$

then we have

$$
f(x, \xi) \leq\left(\lambda_{1}-d\right) \xi+k, \quad x \in \bar{D}, \xi \geq 0 .
$$

We show that the boundary value problem

$$
\begin{cases}A u=\left(\lambda_{1}-d\right) u+k & \text { in } D \\ B u=0 & \text { on } \partial D\end{cases}
$$

has a positive solution $u \in C^{2+\theta}(\bar{D})$.

First it is easy to see that $u \in C^{2+\theta}(\bar{D})$ is a solution of problem (2.3) if and only if it satisfies the following operator equation:

$$
u=\left(\lambda_{1}-d\right) K u+K k \quad \text { in } C_{e}(\bar{D}) .
$$

But we remark that the largest eigenvalue $\left(\lambda_{1}-d\right) / \lambda_{1}$ of the operator $\left(\lambda_{1}-d\right) K$ is less than 1 , and that the function $K k$ is positive on $\bar{D}$. Thus, applying the positivity lemma to our situation, we can find a solution $\psi \in C^{2+\theta}(\bar{D})$ of equation (2.4), or equivalently, a solution of problem (2.3).

Then we have by condition (2.2)

$$
\begin{cases}A \psi-f(x, \psi) \geq\left(\lambda_{1}-d\right) \psi+k-\left(\left(\lambda_{1}-d\right) \psi+k\right)=0 & \text { in } D \\ B \psi=0 & \text { on } \partial D\end{cases}
$$

This proves that the function $\psi \in C^{2+\theta}(\bar{D})$ is a supersolution of problem $(*)$.

(IV) One may assume that the super- and subsolutions $\psi, \phi_{\varepsilon}$ satisfy the condition

$$
\phi_{\varepsilon} \leq \psi \quad \text { on } \bar{D}
$$

Furthermore, if we take a constant $\sigma>0$ such that

$$
\max _{\bar{D}} \phi_{\varepsilon}, \max _{\bar{D}} \psi \leq \sigma
$$

then it follows that the functions $\psi$ and $\phi_{\varepsilon}$ are respectively super- and subsolutions of problem $(*)$ taking values in the interval $[0, \sigma]$.

Therefore our theorem follows from an application of [TU2, Theorem 1].

The proof of Theorem 1 is complete. 


\section{Proof of Theorem 2}

By Theorem 1, it suffices to prove that condition (1.2) is necessary for the existence of positive solutions of problem $(*)$.

We associate with problem $(\dagger)$ an unbounded linear operator $\mathfrak{A}$ from the Hilbert space $L^{2}(D)$ into itself as follows:

(a) The domain of definition $D(\mathfrak{A})$ is the space

$$
D(\mathfrak{A})=\left\{u \in H^{2}(D): B u=0\right\} .
$$

(b) $\mathfrak{A} u=A u, u \in D(\mathfrak{A})$.

Then it is known (cf. [Ta1, Theorems 7.3 and 7.4]) that the operator $\mathfrak{A}$ is a non-negative, self-adjoint operator in $L^{2}(D)$, and has a compact resolvent. Hence we find that the first eigenvalue $\lambda_{1}$ of $\mathfrak{A}$ is characterized by the following formula:

$$
\lambda_{1}=\min \{(\mathfrak{A} u, u): u \in D(\mathfrak{A}),\|u\|=1\}
$$

where $\|\cdot\|$ is the norm on $L^{2}(D)$.

First we show that

$$
\lambda_{1}<\ell
$$

Since the function $f(\xi) / \xi$ is strictly decreasing, it follows that

$$
m<\frac{f(\xi)}{\xi}<\ell, \quad 0<\xi<\infty .
$$

Now let $u \in C^{2}(\bar{D})$ be a positive solution of problem $(*)$ :

$$
\begin{cases}A u=f(u) & \text { in } D, \\ u>0 & \text { in } D, \\ B u=0 & \text { on } \partial D .\end{cases}
$$

Then, since $u \in D(\mathfrak{A})$, we have by inequality (3.3) with $\xi=u(x)$

$$
(\mathfrak{A} u, u)=(A u, u)=\int_{D} f(u) u d x<\ell \int_{D} u^{2} d x
$$

Hence inequality (3.2) follows by using formula (3.1).

Next we show that

$$
\lambda_{1}>m \text {. }
$$

If $u \in C^{2}(\bar{D})$ is a positive solution of problem $(*)$, we let

$$
d=\frac{f\left(\|u\|_{\infty}+1\right)}{\|u\|_{\infty}+1}
$$


where $\|u\|_{\infty}=\max _{\bar{D}} u$. We remark that $d>m$.

Now we consider the eigenvalue problem

$$
\begin{cases}A u-d u=\lambda u & \text { in } D \\ B u=0 & \text { on } \partial D,\end{cases}
$$

and let $\lambda_{1}(d)$ be its first eigenvalue. Then, by formula (3.1), we find that

$$
\lambda_{1}(d)=\min \{((\mathfrak{A}-d I) u, u): u \in D(\mathfrak{A}),\|u\|=1\}=\lambda_{1}-d .
$$

Furthermore, by [Ta2, Theorem 1], one may assume that the first eigenvalue $\lambda_{1}(d)$ has a positive eigenfunction $\varphi \in C^{2+\theta}(\bar{D})$ :

$$
\begin{cases}A \varphi-d \varphi=\lambda_{1}(d) \varphi & \text { in } D, \\ \varphi>0 & \text { in } D, \\ B \varphi=0 & \text { on } \partial D .\end{cases}
$$

Then we have the following:

Claim 3.1. $\lambda_{1}(d)=\lambda_{1}-d>0$.

Proof. Since the function $f(\xi) / \xi$ is strictly decreasing, it follows from formula (3.5) that

$$
f(u(x))>d u(x), \quad x \in D .
$$

Hence we have

$$
(\mathfrak{A} u, \varphi)=(A u, \varphi)=\int_{D} f(u) \varphi d x>d \int_{D} u \varphi d x .
$$

On the other hand, by the self-adjointness $\mathfrak{A}$, it follows that

$$
(\mathfrak{A} u, \varphi)=(u, \mathfrak{A} \varphi)=(u, A \varphi)=\int_{D} u\left(\lambda_{1}(d)+d\right) \varphi d x .
$$

Thus, combining formulas (3.6) and (3.7), we obtain that

$$
\lambda_{1}(d) \int_{D} u \varphi d x>0
$$

This proves Claim 3.1, since we have $u>0, \varphi>0$ in $D$.

Summing up, we have proved that

$$
\lambda_{1}>d>m .
$$

The desired inequality (1.2) follows from inequalities (3.2) and (3.4).

(II) Finally we prove the uniqueness of positive solutions of problem $(*)$ (cf. [BO, Section 2]).

Let $u_{i} \in C^{2}(\bar{D}), i=1,2$, be two positive solutions of problem $(*)$ :

$$
\begin{cases}A u_{i}=f\left(u_{i}\right) & \text { in } D, \\ u_{i}>0 & \text { in } D, \\ B u_{i}=0 & \text { on } \partial D .\end{cases}
$$

The next claim is an essential step in the proof of uniqueness of positive solutions (cf. [BO, Lemma 1]): 
Claim 3.2. $u_{1} / u_{2}, u_{2} / u_{1} \in C(\bar{D})$.

Proof. Since the function $f(\xi) / \xi$ is strictly decreasing, one can find two nonnegative constants $\omega_{i}, i=1,2$, such that

$$
f\left(u_{i}\right)+\omega_{i} u_{i} \geq 0 \quad \text { in } D .
$$

Indeed, it suffices to take

$$
\omega_{i}=\max \left\{0,-\frac{f\left(\left\|u_{i}\right\|_{\infty}\right)}{\left\|u_{i}\right\|_{\infty}}\right\}, \quad i=1,2 .
$$

Then the solutions $u_{i}, i=1,2$, are expressed as follows:

$$
\begin{aligned}
& u_{i}=K_{\omega_{i}}\left(f\left(u_{i}\right)+\omega_{i} u_{i}\right), \\
& f\left(u_{i}\right)+\omega_{i} u_{i} \geq 0 \quad \text { in } D .
\end{aligned}
$$

Here $K_{\omega_{i}}$ is the resolvent of the boundary value problem

$$
\begin{cases}\left(A+\omega_{i}\right) u=\varphi & \text { in } D, \\ B u=0 & \text { on } \partial D .\end{cases}
$$

Hence Claim 3.2 follows from the strong positivity of the resolvents $K_{\omega_{i}}, i=1,2$ (see [TU1, inequality (2.4)]).

By Claim 3.2, we can apply Green's formula to obtain that

$$
\begin{aligned}
& \int_{D}\left(\frac{f\left(u_{1}\right)}{u_{1}}-\frac{f\left(u_{2}\right)}{u_{2}}\right)\left(u_{1}^{2}-u_{2}^{2}\right) d x \\
= & \int_{D}\left(\frac{A u_{1}}{u_{1}}-\frac{A u_{2}}{u_{2}}\right)\left(u_{1}^{2}-u_{2}^{2}\right) d x \\
= & -\int_{D} \sum_{i=1}^{N} \frac{\partial}{\partial x_{i}}\left(\sum_{j=1}^{N} a^{i j} \frac{\partial u_{1}}{\partial x_{j}}\right) u_{1} d x+\int_{D} \sum_{i=1}^{N} \frac{\partial}{\partial x_{i}}\left(\sum_{j=1}^{N} a^{i j} \frac{\partial u_{1}}{\partial x_{j}}\right)\left(\frac{u_{2}^{2}}{u_{1}}\right) d x \\
& -\int_{D} \sum_{i=1}^{N} \frac{\partial}{\partial x_{i}}\left(\sum_{j=1}^{N} a^{i j} \frac{\partial u_{2}}{\partial x_{j}}\right) u_{2} d x+\int_{D} \sum_{i=1}^{N} \frac{\partial}{\partial x_{i}}\left(\sum_{j=1}^{N} a^{i j} \frac{\partial u_{2}}{\partial x_{j}}\right)\left(\frac{u_{1}^{2}}{u_{2}}\right) d x \\
= & \int_{D} \sum_{i, j=1}^{N} a^{i j} \frac{\partial u_{1}}{\partial x_{i}} \frac{\partial u_{1}}{\partial x_{j}} d x-\int_{D} \sum_{i, j=1}^{N} a^{i j} \frac{\partial u_{1}}{\partial x_{i}} \frac{\partial}{\partial x_{j}}\left(\frac{u_{2}^{2}}{u_{1}}\right) d x \\
& -\int_{\partial D} \frac{\partial u_{1}}{\partial \boldsymbol{\nu}} u_{1} d \sigma+\int_{\partial D} \frac{\partial u_{1}}{\partial \boldsymbol{\nu}}\left(\frac{u_{2}^{2}}{u_{1}}\right) d \sigma \\
& +\int_{D} \sum_{i, j=1}^{N} a^{i j} \frac{\partial u_{2}}{\partial x_{i}} \frac{\partial u_{2}}{\partial x_{j}} d x-\int_{D} \sum_{i, j=1}^{N} a^{i j} \frac{\partial u_{2}}{\partial x_{i}} \frac{\partial}{\partial x_{j}}\left(\frac{u_{1}^{2}}{u_{2}}\right) d x \\
& -\int_{\partial D} \frac{\partial u_{2}}{\partial \boldsymbol{\nu}} u_{2} d \sigma+\int_{\partial D} \frac{\partial u_{2}}{\partial \boldsymbol{\nu}}\left(\frac{u_{1}^{2}}{u_{2}}\right) d \sigma .
\end{aligned}
$$


Here we remark that the four integrals over $\partial D$ on the last line of formula $(3.8)$ vanish. Indeed, it suffices to note that

$$
\left|\begin{array}{ll}
\frac{\partial u_{1}}{\partial \boldsymbol{\nu}} & u_{1} \\
\frac{\partial u_{2}}{\partial \boldsymbol{\nu}} & u_{2}
\end{array}\right|=0 \quad \text { on } \partial D
$$

since the solutions $u_{1}$ and $u_{2}$ satisfy the boundary conditions

$$
\left(\begin{array}{ll}
\frac{\partial u_{1}}{\partial \boldsymbol{\nu}} & u_{1} \\
\frac{\partial u_{2}}{\partial \boldsymbol{\nu}} & u_{2}
\end{array}\right)\left(\begin{array}{l}
a \\
b
\end{array}\right)=\left(\begin{array}{l}
0 \\
0
\end{array}\right) \quad \text { on } \partial D
$$

and since $(a, b) \neq(0,0)$ on $\partial D$.

Therefore we find that

$$
\begin{aligned}
& \int_{D}\left(\frac{f\left(u_{1}\right)}{u_{1}}-\frac{f\left(u_{2}\right)}{u_{2}}\right)\left(u_{1}^{2}-u_{2}^{2}\right) d x \\
= & \int_{D} \sum_{i, j=1}^{N} a^{i j}\left(\frac{\partial u_{1}}{\partial x_{i}}-\frac{u_{2}}{u_{1}} \frac{\partial u_{1}}{\partial x_{i}}\right)\left(\frac{\partial u_{1}}{\partial x_{j}}-\frac{u_{2}}{u_{1}} \frac{\partial u_{1}}{\partial x_{j}}\right) d x \\
& +\int_{D} \sum_{i, j=1}^{N} a^{i j}\left(\frac{\partial u_{2}}{\partial x_{i}}-\frac{u_{1}}{u_{2}} \frac{\partial u_{2}}{\partial x_{i}}\right)\left(\frac{\partial u_{2}}{\partial x_{j}}-\frac{u_{1}}{u_{2}} \frac{\partial u_{2}}{\partial x_{j}}\right) d x \\
\geq & 0 .
\end{aligned}
$$

This implies that $u_{1} \equiv u_{2}$ in $D$, since the function $f(\xi) / \xi$ is strictly decreasing.

The proof of Theorem 2 is now complete.

\section{REFERENCES}

[Am] H. Amann, Fixed point equations and nonlinear eigenvalue problems in ordered Banach spaces, SIAM Rev. 18 (1976), 620-709.

[BO] H. Brezis and L. Oswald, Remarks on sublinear elliptic equations, Nonlinear Analysis TMA 10 (1986), 55-64.

[De] D. G. de Figueiredo, Positive solutions of semilinear elliptic problems, Lecture Notes in Mathematics, No. 957, Springer-Verlag, Berlin Heidelberg New York, 1982, pp. 34-87.

[Kr] M. A. Krasnosel'skii, Positive solutions of operator equations, P. Noordhoff, Groningen, 1964.

[SC] R. B. Simpson and D. S. Cohen, Positive solutions of nonlinear elliptic eigenvalue problems, J. Math. Mech. 19 (1970), 895-910.

[Ta1] K. Taira, On some degenerate oblique derivative problems, J. Fac. Sci. Univ. Tokyo Sect. IA 23 (1976), 259-287.

[Ta2] K. Taira, Bifurcation for nonlinear elliptic boundary value problems I, Collectanea Mathematica 47 (1996), 207-229.

[TU1] K. Taira and K. Umezu, Bifurcation for nonlinear elliptic boundary value problems II, Tokyo J. Math. 19 (1996), 387-396.

[TU2] K. Taira and K. Umezu, Bifurcation for nonlinear elliptic boundary value problems III, Adv. Differential Equations 1 (1996), 709-727.

Department of Mathematics, Hiroshima University, Higashi-Hiroshima 739, Japan

Institute of Mathematics, University of Tsukuba, Tsukuba 305, Japan 